\title{
Application d'un modèle simplifié aux zones du Sahel
}

\author{
Application of a simplified model to areas in the Sahel
}

\author{
Georges Girard
}

Directeur de Recherches, ORSTOM

\section{Introduction}

Le présent article apporte une démonstration méthodologique de l'utilité pratique des modèles hydrologiques.

Ils sont les fruits des travaux scientifiques entrepris par les hydrologues de l'ORSTOM pour valoriser l'abondante information hydroclimatologique dont ils disposaient afin de rendre compte des phénomènes engendrés par les aléas climatiques récents dans le Sahel Africain, travaux exécutés dans le cadre d'une convention d'action urgente avec la DGRST.

Le modèle à discrétisation spatiale requérant des données sur le milieu physique souvent indisponibles ne peut être utilisé au maximum de sa puissance sur de très nombreux bassins. Sa fonction de production légèrement modifiée a servi de base à un modèle global, lui plus aisément utilisable.

Nous avons également mis au point un modèle de ruissellement très simplifié pour simuler la lame écoulée journalière d'un petit bassin versant sahélien directement à partir des résultats d'exploitation des bassins représentatifs de l'ORSTOM lorsque les informations sur le milieu physique faisaient totalement défaut. En utilisant les séries pluviométriques aux stations de longue durée, ces modèles permettent de constituer des séries de lames d'écoulement annuelles facilitant l'appréciation de la variabilité interannuelle de la ressource en eau.

La simplicité de ce dernier modèle le rend, sous réserve de certaines contraintes explicitées en fin d'article, applicable à tout bassin représentatif de la zone sahélienne, et le calage des paramètres en est aisé et rapide.

C'est donc un bon outil de l'hydrologie appliquée. Après la présentation des considérations ayant conduit à l'élaboration du modèle simplifié pour le Sahel, nous donnons les résultats de l'application du modèle à divers bassins.
Les figures 1 et 2 situent sur la carte des bassins représentatifs de l'ORSTOM les bassins versants étudiés.

Ces bassins versants sont tous situés dans la région sahélienne moins de $750 \mathrm{~mm}$ de pluviosité annuelle ou légèrement plus au sud jusque vers $850 \mathrm{~mm}$ de pluviosité. Quelques références sont également faites au bassin de Korhogo (nord Côte d'Ivoire, près de $1400 \mathrm{~mm}$ de pluviosité) situé en région tropicale sur lequel des études fines du régime des précipitations ont été effectuées.

\section{Elaboration du modèle simplifié Sahélien}

Diverses considérations ont été faites afin de permettre l'élaboration du modèle simplifié applicable au régime sahélien. Ces considérations portent essentiellement sur les relations hydropluviométriques entre précipitations et hauteurs de lame ruisselée résultantes, c'est-à-dire sur la fonction de production de ce modèle.

L'orientation prise à cette occasion est étroitement conditionnée par le milieu sahélien d'une part et par l'intention d'autre part d'avoir un modèle simple et rustique. L'hypothèse de départ est que la fonction de production ne doit s'appuyer que sur des relations hydropluviométriques établies. Le modèle ne peut en conséquence qu'être global. Destiné à être mis en œuvre sur de faibles surfaces on admet que la hauteur moyenne de précipitation correspond à la hauteur ponctuelle de référence. C'est à partir de la série des précipitations journalières au poste de référence que l'on reconstituera une longue série de lame annuelle écoulée.

\section{Analyse de la précipitation journalière}

On utilise les notions classiques de pluie totale journalière $H t$ en $\mathrm{mm}$ et de pluie utile $H u$ en $\mathrm{mm}$, cette dernière étant la partie de la précipitation totale dont 


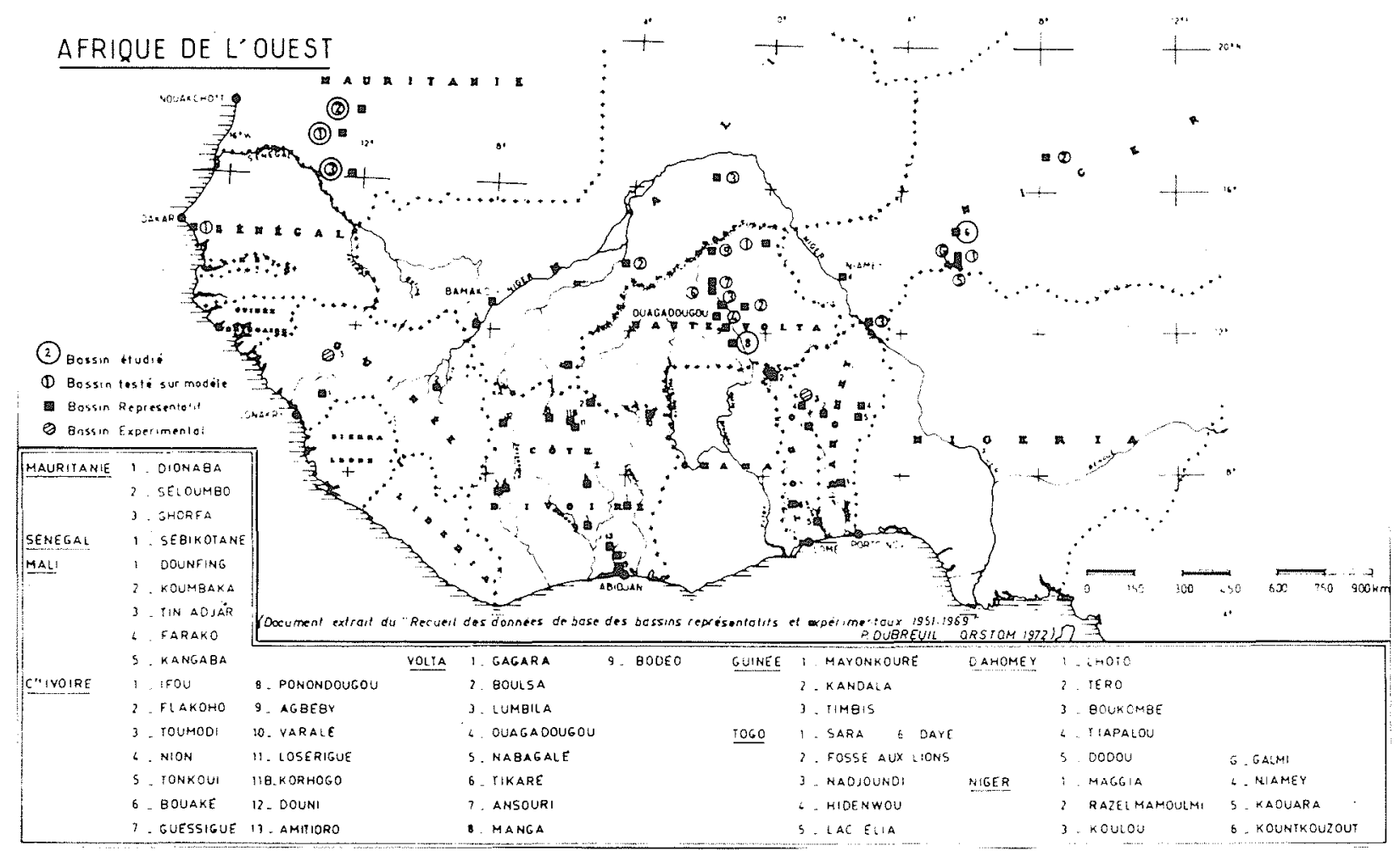

Figure 1. - Afrique de l'Ouest. Situation des bassins représentatifs et expérimentaux

l'intensité était supérieure ou égale à un seuil fixe d'intensité $S$ choisi à priori en considérant la susceptibilité au ruissellement des terrains du bassin versant.

A partir d'échantillons de relevés pluviographiques sur plusieurs années au même poste et en faisant varier le seuil $S$ nous avons trouvé une relation moyenne entre la hauteur utile journalière et la hauteur totale journalière.

Ce travail a été réalisé pour 10 années de données pluviographiques obtenues sur le bassin de Korhogo (Côte d'Ivoire) et donne :

$$
H u=0,62 \quad(H t-5)
$$

C'est-à-dire que la relation obéit à une loi de forme générale:

$$
H u=a \quad(H t-S)
$$

Cette liaison a été trouvée à l'échelle journalière en sommant toutes les pluies et pluies utiles sur de très courtes périodes. Elle existe fondamentalement au niveau de l'averse $\left({ }^{1}\right)$ et se retrouve ici parce que la probabilité d'avoir une averse par jour est très grande et que la forme moyenne de l'averse se conserve dans le cas de plusieurs averses par jour.

L'utilisation de cette liaison moyenne est admissible ici car bien que travaillant sur une base journalière, le modèle n'est envisagé que pour fournir des lames écoulées cohérentes à l'échelle du mois et de l'année.

(1) Si $P$ est la hauteur de l'averse en $\mathrm{mm}$, on trouve pour Korhogo $\mathrm{Hu} u_{c}$ de l'averse $=0,786(P-2)$ pour $C<50 \mathrm{~mm}$.

\section{Relation hydropluviométrique de l'événement averse- crue}

On sait que la plupart des études approfondies sur la forme de la relation entre lame ruisselée et pluie au niveau de l'événement averse-crue réalisée à partir des données de bassins versants représentatifs a permis d'aboutir à l'idée d'une forme parabolique. L'asymptote de celle-ci représente en quelque sorte l'enveloppe supérieure de la relation et exprime implicitement l'idée d'un coefficient de ruissellement maximal constant.

C'est sur cette idée que nous avons modelé la fonction de production à élaborer. Sur le bassin de Korhogo où les événements averse-crue sont en nombre supérieur à 100 , on a vérifié que tous les points dans un plan lame ruisselée-hauteur utile s'inscrivaient entre deux droites d'équation

$$
L R_{c}=0,43\left(H u_{c}-S u\right) \quad L R_{c}=\underset{\substack{\text { lame ruisselée d'une } \\ \text { crue en mm }}}{\text { mand }}
$$

la droite inférieure correspondant à une saturation préalable des terrains à son niveau minimal $(S u=50 \mathrm{~mm})$; la droite supérieure au niveau maximal de saturation $(S u=0)$

On pose donc en principe que la relation hydropluviométrique à l'échelle journalière est de la forme :

$$
L R=K(H u-S u)
$$

le paramètre $K$ ressemblant à un coefficient de ruissellement et $S u$ caractérisant l'état de saturation préalable 


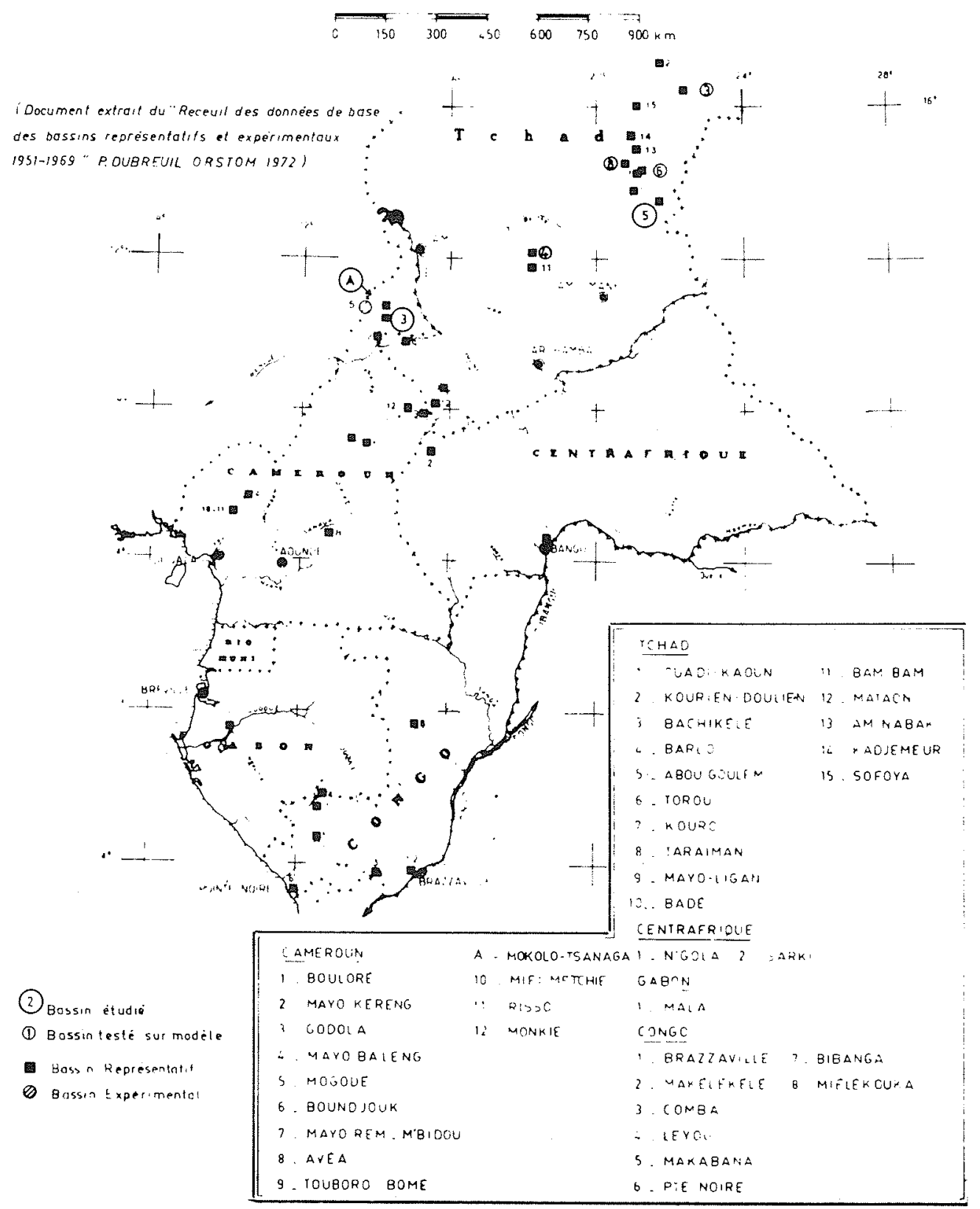

Figure 2. - Afrique Centrale. Situation des bassins représentatifs et expérimentaux

du bassin. En associant les équations (1) et (2) on obtient une relation de la forme:

$$
L R=K o(H t-H o)
$$

On a voulu vérifier si cette relation (3) apparemment simpliste était réaliste pour les zones sahéliennes. Elle a été recherchée et trouvée pour les 33 bassins versants répertoriés sur les figures 1 et 2 .

Bien entendu, elle a été établie au niveau de l'événement averse-crue et à partir de $\mathrm{Ht}_{c}$ (hauteur totale de l'averse). Il est apparu que la valeur minimale de $H o$ était nulle dans la majorité des cas et n'excédait pas $4 \mathrm{~mm}$ dans les rares autres cas. On a donc convenu qu'une valeur minimale nulle simplifierait l'utilisation de la relation (3).

Le paramètre $H o$ varie donc entre 0 et $H O_{\max }$, valeur de référence de l'état de saturation des terrains. Les va- leurs de $\mathrm{Ko}$ et $\mathrm{Ho}_{\max }$ pour tous les bassins étudiés sont réunies dans le tableau 1.

Grâce à l'observation simultanée d'une gamme imposante de couples averse-crue dont certains de fréquence rare, le diagramme $(L R-H T)$ pour le bassin versant de Djajibine (Oued Ghorfa) présente deux "rails" parfaitement bien définis (Fig. 3)

On peut expliquer cette liaison quasi linéaire entre la lame ruisselée et la hauteur de l'averse pour ce bassin sans détruire les principes même de la théorie de l'infiltration, de la manière suivante :

Lorsque la hauteur de l'averse croît, la durée totale de l'averse croît également et la durée de la pluie utile, définie pour un seuil donné d'intensité, croît proportionnellement en moyenne avec la hauteur de l'averse. Si ce seuil d'intensité correspond à la valeur de la perméabilité moyenne, il est tout à fait normal que la pluie excéden- 


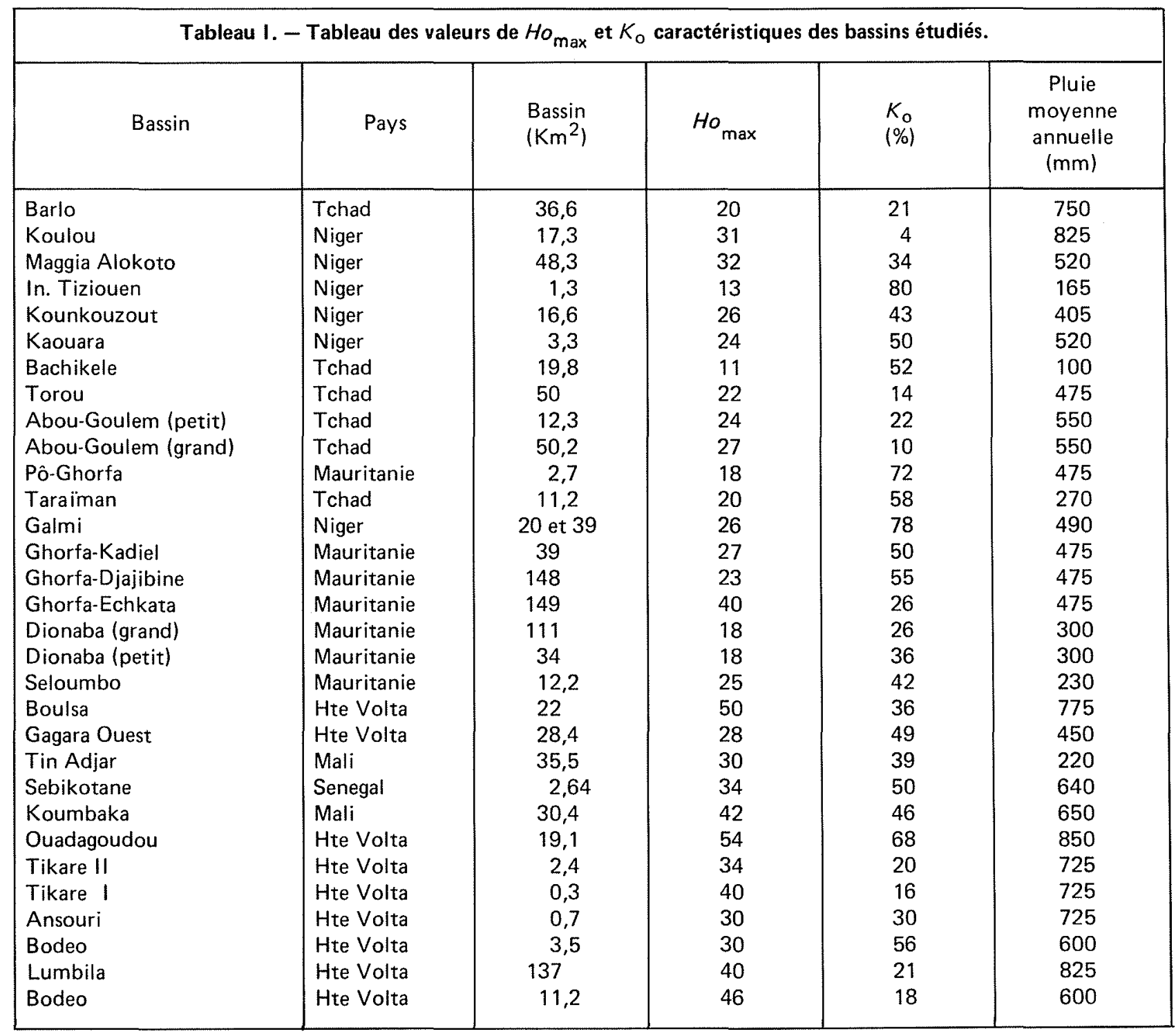

taire, donc la lame ruisselée, varie linéairement-avec la hauteur de l'averse.

L'expérience montre que la relation (3) $\mathrm{Lr}-\mathrm{Ht}$ est bien linéaire même pour des averses exceptionnellement très fortes, malgré la perméabilité des sols d'un bassin qui varie considérablement d'un point à un autre selon la nature du sol, la couverture végétale et l'utilisation de ces sols, sans oublier l'état initial d'humidité de ceux-ci.

Nous devons considérer comme très importante cette constatation expérimentale qui tend à montrer l'influence extrêmement grande de l'homogénéisation spatiale.

A l'échelle de l'averse, la loi expérimentale liant $L r$ à $H U$ présente une très grosse dispersion due en grande partie à la variabilité des intensités de l'averse pour un même total pluviométrique et à la variabilité de l'état antérieur de saturation du bassin versant.

Cette dernière influence s'estompe pour un bassin versant présentant en moyenne une imperméabilité notoire. C'est alors que la dispersion des points dans le diagramme $(L R-H U)$ résulte directement des caracté- ristiques propres à l'averse. C'est le cas du petit bassin du Pô sur l'Oued-Ghorfa pour lequel nous avons noté une dispersion comparable sur les diagrammes $(L R-H T)$ et $(H U-H T)$. Pour ce bassin très imperméable, à l'exception d'une partie du bassin ne contribuant pas, semble-t-il, à l'écoulement (20\%), partie d'ailleurs reconnue par les hydrologues sur le terrain, la pente $K o$ des droites limites de la bande est de 0,80 . Cette pente, pour la même région climatologiquement homogène va diminuer quand la perméabilité moyenne des sols et du sous-sol du bassin va augmenter.

C'est ainsi que pour les bassins de Seloumbo, l'OuedMoktar et l'Oued-Ali, dont le sous-sol est plutôt gréseux, la pente des "rails" passe à des valeurs de 0,56 et de 0,42 et l'écartement de ces "rails" devient plus important. Il est tout à fait probable que les variations totales d'humidité dans tous les sols de ce bassin soient plus importantes que celles du bassin du Pô pour lequel l'écartement des "rails" est beaucoup plus faible.

En conclusion, nous dirons que la pente des "rails" $K_{0}$ est liée à l'extension des zones à rétention définitive 

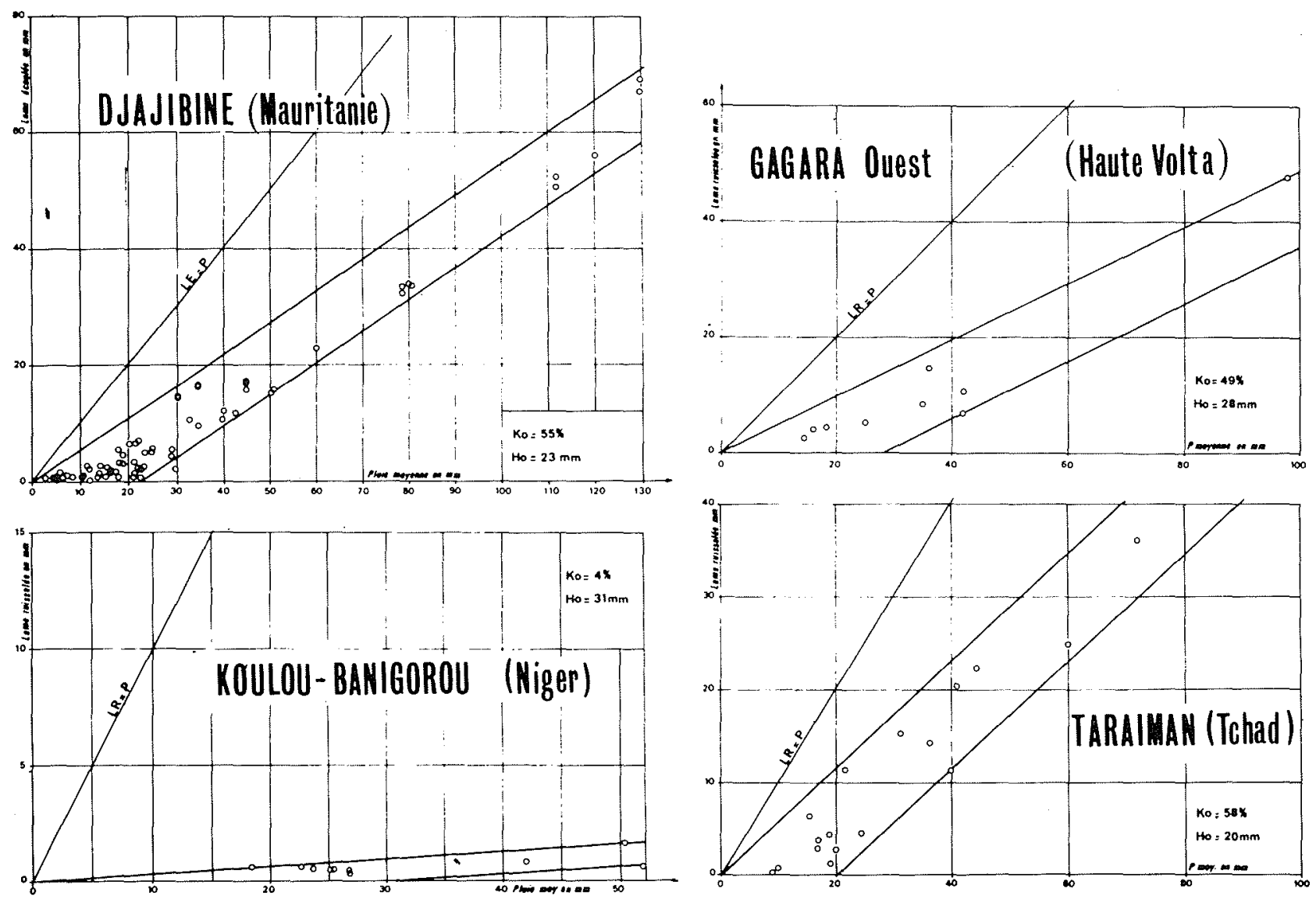

Figure 3

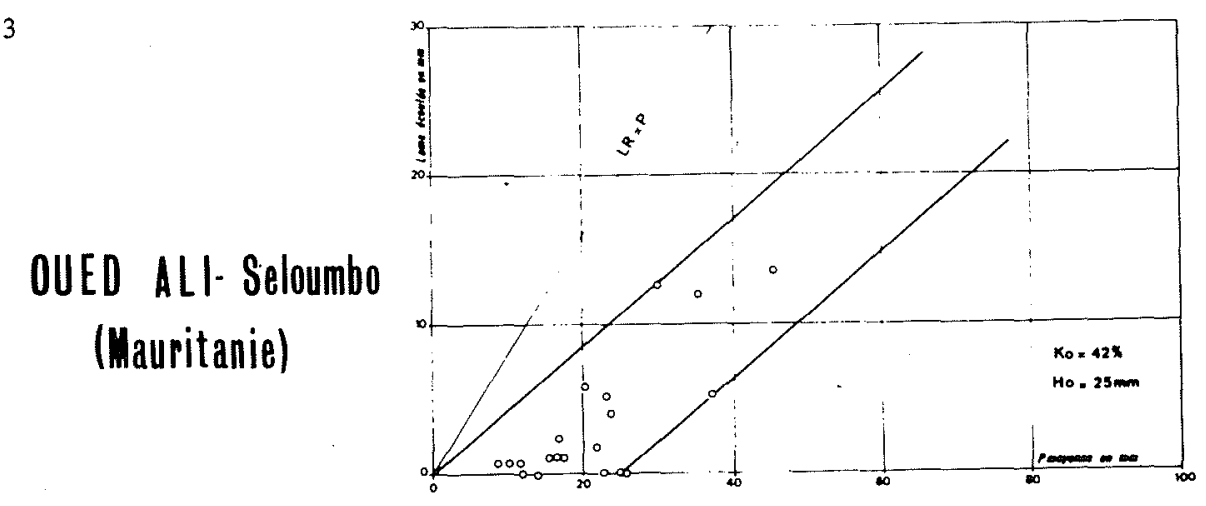

Figure 4

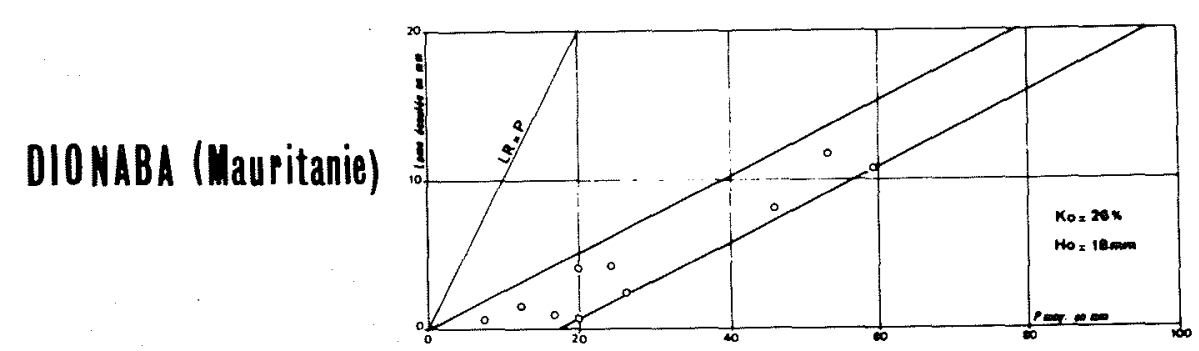

Figure 5

Relation lame écoulée ou ruisselée. Pluie moyenne d'une averse 
du bassin, à la valeur de la perméabilité et à la dimension $\mathrm{du}$ bassin versant. Cette pente décroît avec l'extension des zones de rétention, l'accroissement de la perméabilité et l'augmentation de la superficie du bassin.

L'écartement $H o_{\max }$ des "rails" dépend de la capacité de rétention des sols, de l'amplitude de variation annuelle du stock d'eau des sols et de la structure des précipitations (fréquence et intensités). On voit ainsi $\mathrm{Ho}_{\mathrm{max}}$ très faible et égal à $18 \mathrm{~mm}$ pour le petit bassin imperméable du Pô-Ghorfa, atteindre $25 \mathrm{~mm}$ pour celui plus grand de l'Oued Ali sur terrains gréseux et finir avec des valeurs voisines de $50 \mathrm{~mm}$ pour tous les bassins recouverts de sols relativement profonds et dotés d'une certaine rétention (Boulsa, Ouagadougou) (Fig. 3 à 5).

Comment prendre en compte la variabilité du paramètre $H o$ dans la relation (3) qui est somme toute à elle seule la fonction de production du modèle simplifié sahélien?

La variation de $H_{0}$ pour un même bassin versant en liaison avec l'état d'humidité de celui-ci ne peut être qu'approximativement évaluée.

Si nous notons parfaitement bien une tendance nette de la variation de $H_{0}$ avec la succession des précipitations dans le temps compte tenu de leur importance, nous n'avons pas à l'heure actuelle recherché de relation expérimentale mais seulement admis une hypothèse sur la forme de cette relation possible.

On adopte pour calculer $H_{0}$ une fonction de la forme

$$
H j+1=(H j+H t j) C
$$

dans laquelle

$H$ tj est la pluie moyenne totale du jour $J$

$H j$ un index des pluies antérieures à $J$

$H j+1$ l'index des pluies antérieures à $J+1$. .

$C$ un coefficient compris entre 0 et 1 et souvent voisin de 0,7

Cette équation (4) permet de calculer un index $H$ dont on tire la valeur de $H_{0}$ pour le même jour $J$ de la manière suivante :

a) si $H j>H_{\max } \quad H o=0$

b) si $H j=0 \quad H o=H o_{\max }$

c) si $0<H j<H o_{\max } \quad H o=H o_{\max }-H j$

Le coefficient $C$ est un paramètre de réglage du modèle.

\section{Influence de la répartition et de la forme des phies dans une journée}

L'utilisation d'une relation $L r H t$ (3) établie à l'échelle de l'événement averse-crue est admissible à l'échelle journalière en pays sahélien où il y a sûrement une très forte probabilité à n'avoir qu'une pluie par jour. Quant à la forme et à l'intensité de l'averse, leur rôle est confondu dans ce modèle, à l'intérieur du terme $H o$, avec l'influence de la saturation des terrains. Reprenons les deux droites limites de l'équation (3) pour Ho nul ou égal à $H o_{\max }$.

$\mathrm{Si}$ tous les points se groupent entre deux droites bien délimitées, l'écartement horizontal de ces deux droites limites doit être imputé non pas seulement à l'état antérieur d'humidité du bassin mais aussi à la variation de la durée et de l'intensité de la précipitation en cause. Pour une averse de faible intensité et de longue durée, la lame ruisselée sera très faible étant donné qu'une grosse partie de la pluie aura pénétré dans le sol.

Mais comme très souvent la hauteur de l'averse est liée à l'intensité, on peut admettre qu'une relation moyenne existe. Si le bassin versant est en moyenne beaucoup plus imperméable, l'écartement horizontal de ces droites diminue, ce que l'on observe bien sur tous les graphiques (Kadiel $\mathrm{Po}, \ldots$ ).

Pour le bassin du Po (région sahélienne de Mauritanie) la hauteur utile de l'averse pour un seuil de $18 \mathrm{~mm} / \mathrm{h}$ est liée à la hauteur de pluie par la relation $H u_{c}=0,87(H c-4)$ et la lame ruisselée est liée à la hauteur utile par la relation :

$$
\begin{aligned}
& L R_{c}=0,80\left(H u_{c}-H o\right) \\
& H o \text { variant de } 4 \text { à } 14 \mathrm{~mm} .
\end{aligned}
$$

Dans cette région où l'on a en général une seule averse par jour de pluie ayant comme caractéristique d'avoir une pointe d'intensité unique, ces relations ne surprennent pas.

\section{Applications du modèle simplifié}

Le modèle simplifié sahélien a été appliqué à cinq bassins représentatifs de l'ORSTOM :

1. Le bassin de Dionaba (bassin du Gorgol Blanc, Mauritanie)

2. Le bassin de Kadiel, partie de l'ensemble de l'Oued Ghorfa (Mauritanie)

3. Le bassin de l'Oued Ali, partie du bassin de Seloumbo (Mauritanie)

4. Le bassin de Kounkouzout (bassin de Batha, Tchad). 5. Le bassin d'Abougoulem (bassin du Batha, Tchad).

La superficie de ces bassins versants reste comprise entre $10 \mathrm{~km}^{2}$ et $110 \mathrm{~km}^{2}$. Les résultats obtenus peuvent être transposables aux bassins versants plus petits sans restriction et à ceux plus grands mais dont la superficie ne dépasse pas $150 \mathrm{~km}^{2}$, à condition toutefois que la dégradation du réseau hydrographique ne soit pas trop avancée.

\section{Application du modèle simplifié au bassin de Dionaba (Mauritanie)}

Ce bassin a une superficie de $111 \mathrm{~km}^{2}$.

\section{Calage du modèle}

Les deux coefficients du modèle simplifié (Ko, $H o_{\max }$ ) ont été obtenus à partir de la liaison établie entre la lame écoulée et la pluie moyenne sur le bassin versant à la fin des deux campagnes hydrologiques de 1958 et 1959. Les données observées ont été présentées par Y. Brunet-Moret (1960) (Fig. 5).

Un seul calage fut nécessaire pour $C$ puisque la correspondance entre les lames journalières observées et calculées présentait, pour ces deux années 1958 et 1959 , un très grand degré de confiance à l'échelle journalière.

\section{Résultats obtenus}

Notons que pour la station pluviométrique de référence (Moudjeria) la pluviométrie annuelle calculée pour 


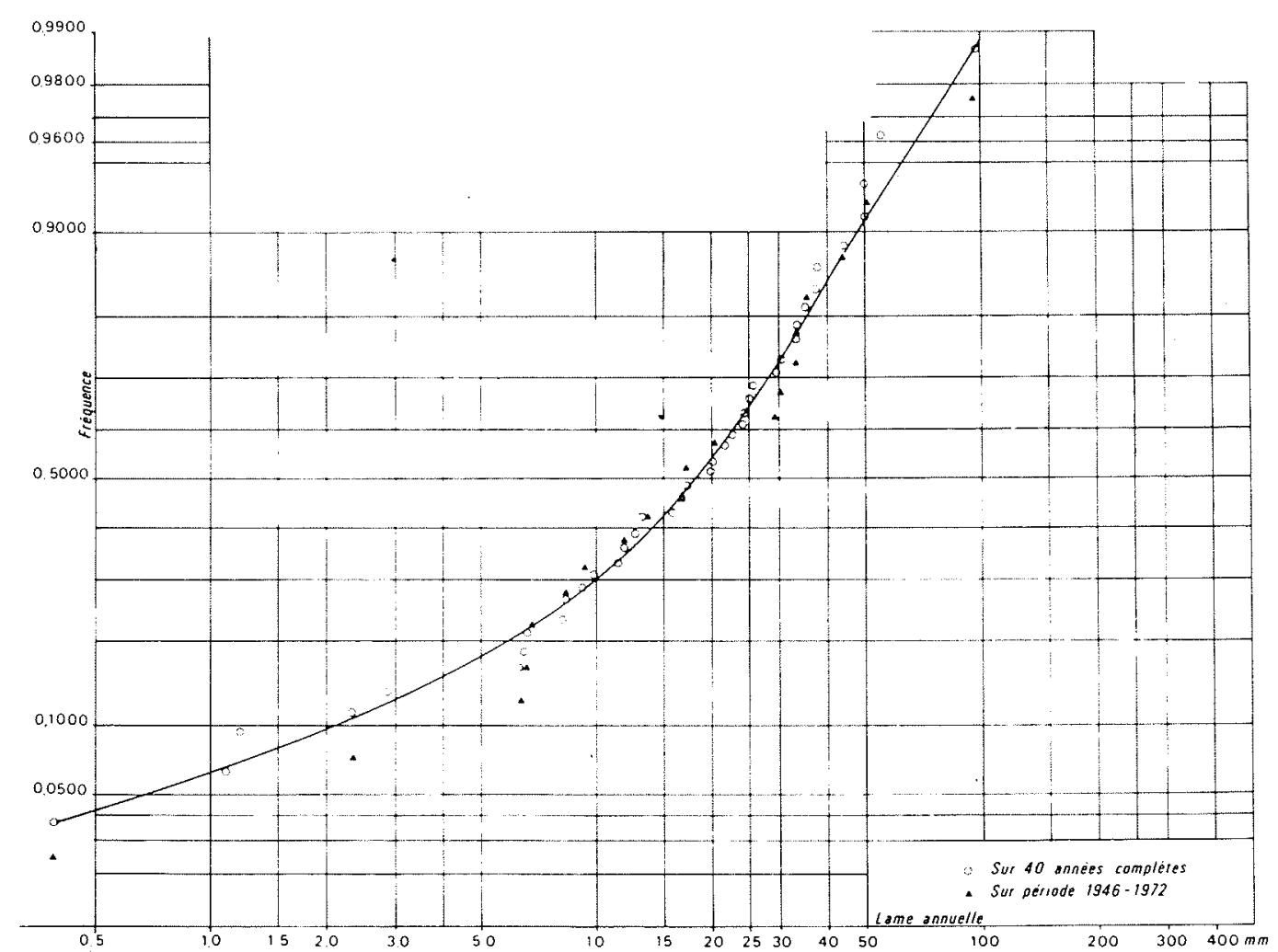

Figure 6. - Distribution des lames annuelles simulées sur le bassin de Dionaba aval à partir du poste de Moudjeria (Mauritanie)

les 16 années complètes de la période 1921-1941 est de $201 \mathrm{~mm}$ et que celle calculée pour les 16 années complètes de la période 1950-1972 atteint $257 \mathrm{~mm}$.

De ce fait, nous avons considéré d'une part la série hydrologique des lames écoulées calculée à partir de 40 années complètes et d'autre part celle correspondant aux 20 dernières années (1946 à 1972) pour montrer la faible différence obtenue sur la distribution statistique de ces résultats.

La dimension réduite de l'échantillon ne permet pas de tirer des conclusions très nettes. Cependant il est possible de montrer que les lames pour les années faibles se trouvent surestimées pour la série complète (cf. Fig. 6).

Les caractéristiques des lames annuelles simulées sont : Lame moyenne

Lame médiane

Lame décennale sèche

Lame décennale humide

Coefficient de variation

$=22,0 \mathrm{~mm}$

$=18,0 \mathrm{~mm}$

$=2 \mathrm{~mm}$

$=49 \mathrm{~mm}$

$=0,87 \mathrm{~mm}$

$=2 \%$

Coefficient d'écoulement décennal humide $=13,5 \%$

Coefficient d'écoulement décennal médian $=8,5 \%$

Application du modèle simplifié au bassin versant de Kadiel, sous bassin de l'Oued Ghorfa (Mauritanie)

Ce bassin a une superficie de $39 \mathrm{~km}^{2}$.

\section{Calage du modèle}

Il suffit de fournir les données des précipitations journalières à une ou plusieurs stations de référence pour obtenir une simulation des débits journaliers sur le bassin versant de Kadiel (République Islamique de Mauritanie) de superficie $39 \mathrm{~km}^{2}$.

\section{Les résultats}

Il est très intéressant de montrer à la fois la souplesse du modèle et l'homogénéité du régime des pluies dans

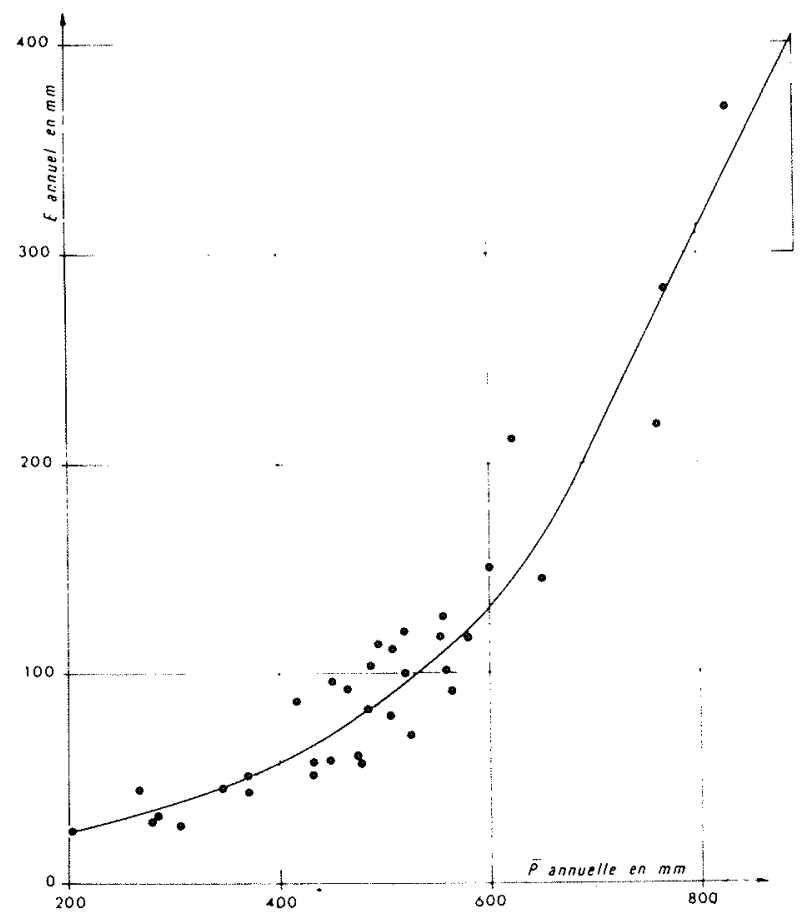

Figure 7. - Ecoulement annuel simulé en fonction de la pluie annuelle à Madaoua (Niger) bassin de Kadiel 


\begin{tabular}{|c|c|c|c|c|}
\hline \multirow{2}{*}{$\begin{array}{l}\text { Valeurs calculées à partir des données de : } \\
\text { Lame moyenne annuelle écoulée }\end{array}$} & $\begin{array}{l}\text { Kankossa } \\
\text { (20 années) }\end{array}$ & \multicolumn{3}{|c|}{$\begin{array}{l}\text { De M'Bout et Madaqua } \\
\text { (38 années) | (36 années) }\end{array}$} \\
\hline & $83 \mathrm{~mm}$ & $\mathrm{~mm}$ & 102 & $\mathrm{~mm}$ \\
\hline Lame annuelle décennale sèche écoulée & $\mathrm{mm}$ & $\mathrm{mm}$ & 30 & $\mathrm{~mm}$ \\
\hline Lame annuelle décennale humide écoulée & $142 \mathrm{~mm}$ & $160 \mathrm{~mm}$ & 200 & $\mathrm{~mm}$ \\
\hline Coefficient de variation des lames écoulées & $0,51 \mathrm{~mm}$ & $0,55 \mathrm{~mm}$ & 0,7 & $2 \mathrm{~mm}$ \\
\hline Lame médiane calculée & $78 \mathrm{~mm}$ & $84 \mathrm{~mm}$ & 90 & $\mathrm{~mm}$ \\
\hline Lame maximale journalière calculée & $35,6 \mathrm{~mm}$ & $45,6 \mathrm{~mm}$ & 32,7 & $\mathrm{~mm}$ \\
\hline Pluie maximale journalière observée & $111,0 \mathrm{~mm}$ & $132,0 \mathrm{~mm}$ & 98,6 & $\mathrm{~mm}$ \\
\hline Pluie annuelle moyenne & $418 \mathrm{~mm}$ & 423 & 484 & $\mathrm{~mm}$ \\
\hline Pluie annuelle maximale & $\mathrm{mm}$ & $\mathrm{mm}$ & 830 & $\mathrm{~mm}$ \\
\hline Pluie annuelle minimale & $\mathrm{mm}$ & $\mathrm{mm}$ & 205 & $\mathrm{~mm}$ \\
\hline
\end{tabular}

Tableau II. - Lames annuelles simulées sur le bassin de Dionaba (Mauritanie) obtenues à partir du poste pluviométrique de Moudjeria

\begin{tabular}{|c|c|c|}
\hline Année complète & Pluie & Lame écoulée \\
\hline 1923 & 233,1 & 24,4 \\
\hline 1924 & 122,0 & 22,9 \\
\hline 1925 & 188,7 & 9,8 \\
\hline 1926 & 112,0 & 1,2 \\
\hline 1927 & 240,4 & 20,1 \\
\hline 1928 & 173,6 & 8,2 \\
\hline 1929 & 215,3 & 15,8 \\
\hline 1930 & 169,5 & 24,2 \\
\hline 1931 & 156,9 & 25,8 \\
\hline 1932 & 248,0 & 37,9 \\
\hline 1933 & 373,3 & 55,7 \\
\hline 1934 & 145,0 & 11,5 \\
\hline 1935 & 282,9 & 25,1 \\
\hline 1936 & 295,0 & 37,9 \\
\hline 1940 & 202,7 & 12,6 \\
\hline 1941 & 55,1 & 0 \\
\hline 1942 & 89,5 & 1,1 \\
\hline 1943 & 403,3 & 50,2 \\
\hline 1944 & 234,5 & 22,6 \\
\hline 1945 & 223,3 & 21,7 \\
\hline 1946 & 118,6 & 8,3 \\
\hline 1947 & 251,7 & 33,6 \\
\hline 1948 & 187,8 & 17,2 \\
\hline 1949 & 196,3 & 13,1 \\
\hline 1950 & 282,0 & 20,1 \\
\hline 1951 & 241,8 & 11,9 \\
\hline 1952 & 291,9 & 29,7 \\
\hline 1953 & 174,8 & 6,6 \\
\hline 1954 & 354,6 & 44,6 \\
\hline 1955 & 387,7 & 35,3 \\
\hline 1957 & 250,0 & 30,3 \\
\hline 1961 & 94,0 & 0,04 \\
\hline 1965 & 204,8 & 9,2 \\
\hline 1966 & 325,2 & 33,3 \\
\hline 1967 & 354,1 & 50,6 \\
\hline 1968 & 153,4 & 6,4 \\
\hline 1969 & 535,0 & 96,5 \\
\hline 1970 & 238,0 & 16,5 \\
\hline 1971 & 159,0 & 6,5 \\
\hline 1972 & 66,5 & 2,3 \\
\hline
\end{tabular}

la bande sahélienne. A cet effet, on a simulé les écoulements du bassin de Kadiel à partir du poste de Madaoua, au Niger, de pluviosité comparable à celle de M'Bout. Le résultat sous la forme de la liaison entre lame écoulée annuelle et hauteur annuelle de pluie est donné pour les données de Madaoua utilisées (Fig. 7).

Des valeurs décennales sèches et humides d'environ $30 \mathrm{~mm}$ et $150 \mathrm{~mm}$ sont tout à fait acceptables pour l'écoulement du bassin versant de Kadiel.

La meilleure reconstitution reste celle de M'Bout. Elle a permis de dresser la distribution statistique des lames calculées.

\section{Les applications du modèle simplifié aux autres bassins}

- Oued Ali sous bassin de l'Oued Seloumbo et du Gorgol Blanc (République de Mauritanie)

- de Kounkouzout (République du Niger)

- d'Abougoulem (République du Tchad)

ont été réalisées sans difficulté notable ; il est à noter que le calage est réalisé à partir du moment où la distribution des écarts journaliers dans un sens ou dans l'autre reste normale. Ces écarts ne dépassent généralement pas $30 \%$.

\section{Conclusion}

Dans les zones sahéliennes, où la densité des stations pluviographiques ne dépasse pas un appareil pour $100000 \mathrm{~km}^{2}$ ou plus, et la période d'observation est très réduite, il était hors de question pour satisfaire la demande d'utiliser les informations fragmentaires sur les intensités des précipitations. Seules les données pluviométriques journalières pouvaient être utilisées dans cette étude qui montre la fiabilité de la méthode appliquée dans cette zone.

Par ailleurs, il a été montré la nécessité de collecter des données hydropluviométriques de bonne qualité et de mettre en place des fichiers opérationnels. 
Tableau III. - Lames annuelles simulées sur le bassin de Kadiel à partir de trois postes pluviométriques $(\mathrm{mm})$.

\begin{tabular}{|c|c|c|c|c|c|c|c|}
\hline Année & M'Bout & Kankossa & Madaoua & Année & M'Bout & Kankossa & Madaoua \\
\hline 1921 & 98,0 & & & 1947 & 65,4 & & 92,5 \\
\hline 1922 & & & & 1948 & 62,3 & & 87,2 \\
\hline 1923 & 55,6 & & & 1949 & 65,4 & & 29,8 \\
\hline 1924 & 204,2 & & & 1950 & 162,8 & & 368,7 \\
\hline 1925 & 41,3 & & & 1951 & 194,7 & & 101,2 \\
\hline 1926 & 22,3 & & & 1952 & 112,2 & & 218,5 \\
\hline 1927 & 62,4 & & & 1953 & 51,0 & 119,0 & 70,2 \\
\hline 1928 & 41,4 & & & 1954 & 125,2 & 140,8 & 145,4 \\
\hline 1929 & 52,5 & & & 1955 & 112,2 & 36,9 & 57,2 \\
\hline 1930 & 143,8 & & & 1956 & 231,4 & 99,1 & 111,5 \\
\hline 1931 & 20,8 & & & 1957 & 113,4 & 77,3 & 57,7 \\
\hline 1932 & & & & 1958 & 77,7 & 154,4 & 116,8 \\
\hline 1933 & & & & 1959 & 102,6 & 75,8 & 119,6 \\
\hline 1934 & & & & 1960 & 211,4 & 32,4 & 95,9 \\
\hline 1935 & & & & 1961 & 88,7 & 35,2 & 212,2 \\
\hline 1936 & & & 283,6 & 1962 & 79,4 & 105,8 & 100,3 \\
\hline 1937 & & & 117,5 & 1963 & 70,8 & 66,4 & 45,3 \\
\hline 1938 & & & & 1964 & 123,3 & 103,9 & 28,0 \\
\hline 1939 & & & 91,4 & 1965 & 80,1 & 142,6 & 44,2 \\
\hline 1940 & & & 52,1 & 1966 & 50,3 & 76,3 & 104,4 \\
\hline 1941 & & & 79,9 & 1967 & 97,0 & 141,6 & 51,1 \\
\hline 1942 & & & 31,5 & 1968 & 26,1 & 43,2 & 25,9 \\
\hline 1943 & & & 149,9 & 1969 & 64,9 & 102,0 & 58,5 \\
\hline 1944 & 143,3 & & 83,1 & 1970 & 97,3 & 45,8 & 114,6 \\
\hline 1945 & 112,6 & & 126,6 & 1971 & 83,9 & 35,5 & 45,3 \\
\hline 1946 & 77,1 & & 60,9 & 1972 & & 26,7 & \\
\hline
\end{tabular}

\section{Discussion}

Le Président. - Je tiens, M. GIRARD, à vous remercier à plusieurs titres.

D'abord, vous nous avez montré comment on peut tirer parti et utiliser les données de bassins versants représentatifs ; de ce point de vue, votre travail est assez exemplaire. Vous avez véritablement fait une synthèse et montré comment on pouvait transposer les résultats de tels bassins.

Vous donnez à ce propos un exemple assez frappant puisque, pour estimer des lames d'eau dans un bassin versant de Mauritanie, vous prenez les pluies d'un poste au Niger et vous en donnez une justification climatique par la morphologie des averses.

Il y a un autre point que je voudrais souligner et qui pourrait d'ailleurs être la conclusion de notre matinée.

Vous insistez, à juste titre, sur les problèmes de qualité de données hydropluviométriques et de la mise en place de fichiers opérationnels.

Il est évident que les travaux d'élaboration de relations pluiedébit ne seront utiles pour la prévision que dans la mesure où l'on dispose effectivement de données fiables et représentatives.

A l'heure actuelle on parle beaucoup d'utiliser ces relations pluie-débit à ce que l'on appelle la gestion des eaux.

Parler de la gestion des eaux c'est bien, mais il faut avant tout faire des mesures.
Nous avons tous connu des ingénieurs et des techniciens qui ont consacré tout ou partie de leur vie professionnelle à exploiter des réseaux de mesures, que ce soient des réseaux hydrométriques ou des réseaux météorologiques. Si nous pouvons faire des travaux, tels que ceux présentés, aujourd'hui, c'est aux qualités de ces gestionnaires qu'on le doit.

Mais, ici, je ne peux m'empêcher d'être inquiet pour l'avenir, car il semble bien que s'instaure une certaine désaffection pour l'exploitation des réseaux de mesures, aussi bien pour la pluviométrie que pour l'hydrométrie : un peu partout on considère qu'il s'agit d'une tâche triviale, dépourvue de noblesse ou même d'intérêt.

Je crois sérieusement qu'il faudrait inverser la tendance et je souhaiterais que des responsables des Agences de bassins, de l'Administration, des différents services publics ou nationaux concernés, prennent conscience que l'avenir, en ce qui concerne les progrès en matière de gestion et d'utilisation rationnelle des ressources en eau, passe de façon absolue par une revalorisation de cette fonction de gestionnaire des réseaux de mesures hydrométéorologiques.

Messieurs, je vous remercie beaucoup de votre collaboration et je lève la séance.

La séance est levée à 12 heures. 